\title{
The Research of Intelligent Image Recognition Technology Based on Neural Network
}

\author{
LI Huanliang ${ }^{1, a}$ \\ ${ }^{1}$ Yishui Campus of Linyi University, Yishui 276400,China \\ alihuanliang@qq.com
}

Keywords: Image Recognition, Neural Network, Image Processing

\begin{abstract}
Image processing and recognition is carried out on the actual image transformation and transformation so as to achieve the aim of identification. Because of the characteristic of the image information is that it is a two-dimensional space, so the amount of information it contains is very large. Neural network image recognition technology is the modern computer technology, image processing, artificial intelligence, pattern recognition theory developed a new kind of image recognition technology. Before the image recognition need to use digital image processing techniques for image preprocessing and feature extraction. With the theory of artificial intelligence research and the development of computer technology, the application of neural network in image pattern recognition research is increasingly active.
\end{abstract}

\section{Introduction}

The image recognition technology is closely related to social life, image recognition technology is an important branch of computer vision, neural network image recognition technology is along with the modern computer technology, image processing, artificial intelligence, and pattern recognition theory developed a new kind of image recognition technology [1]. To realize the recognition of images, the first to get corresponding image by image acquisition device, so that the digital image; Then the image recognition, and its various information. In this paper, neural network is used to analyze the acquired digital image recognition, the BP neural network is introduced into image recognition field, and combined with conventional digital image processing technology, find out a kind of strong accuracy plane image recognition method [2].

Image recognition involves a lot of information operation, requiring high processing speed and recognition precision, real-time and fault-tolerance of the neural network in accordance with the requirements of image recognition. At first, this paper analyzes the traditional image recognition method, aiming at the limitations of traditional methods, and the complex situations such as images show different state, in the process of image processing algorithm for the image segmentation study and its improvement; At the same time, according to local minimum value of the problems existing in the BP neural network, improve the efficiency of the network, improve the precision of image recognition, and in the process of network training using the method of adaptive learning rate change, reduces the network number of training and the training time. Using the improved BP neural network algorithm for rotational distortion image positioning and recognition, the improved algorithm will be combination of additional momentum and adaptive learning rate, effectively suppress the network into a local minimum point, improves the training speed of network. Finally validated through the experiment, proved the feasibility and effectiveness of the optimization method, and realized by programming, achieve better results.

\section{Summary of the BP neural network}

BP network is multilayer feed forward neural network based on BP algorithm, it is D.E.Rumelhart, J.L.McCellnad and their team studied and designed in 1986 [3]. At present, in the practical application of artificial neural network, the vast majority of the neural network model is the variation of the BP network and its form, it is also a former core to the network, and embodies the 
most essence part of the artificial neural network. BP neural network is a kind of multilayer forward network, consists of input layer and output layer, hidden layer or multilayer structure, a kind of typical three layer BP neural network model is shown in figure 1. The transformation function of hidden layer in general as a nonlinear function. Transform functions of output layer can be nonlinear, can also be linear, by the input, output mapping needs [4].

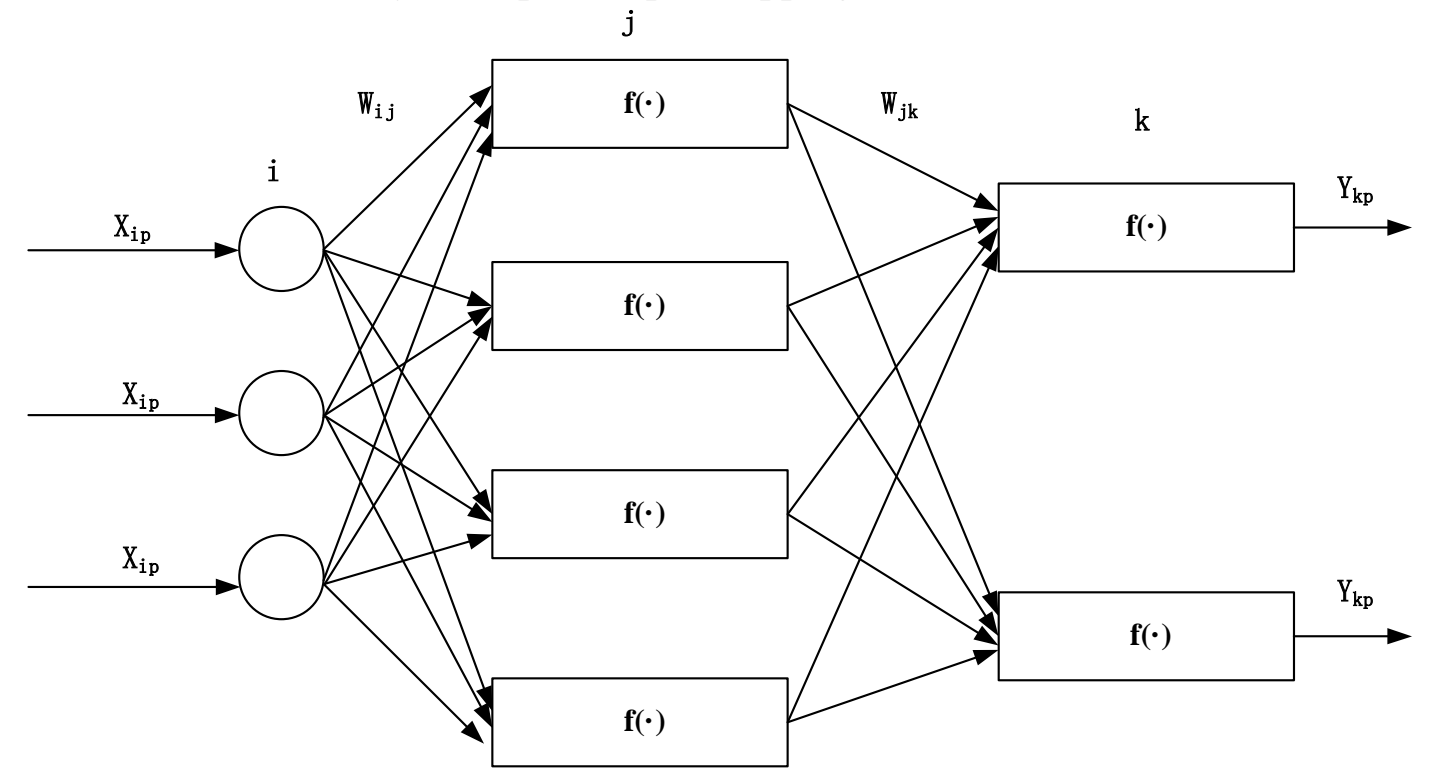

Figure 1. Typical BP neural network model

Back propagation algorithm is the main idea of the learning process is divided into two stages: stage 1 (positive propagation), input information from the input layer to start step by step calculating actual output values of each unit of each layer of neurons state only affect the next layer of neurons state; Phase 2 (back propagation) process, if the output layer fails to achieve the desired output values, the layered recursive calculation of the difference between actual output and desired output value, according to the error correction layer weights before make the error signal to a minimum. By continuously in the direction of the error function is relative to the slope down on network weights and deviation change and approaching the goal. Every weight value and the error change proportional to the effect of network error.

\section{Intelligent image recognition process analysis}

Image recognition as a branch of image technology has been a hot research topic in the field of image processing and pattern recognition. Traditional pattern recognition methods applied to the color of the image recognition is mainly based on image features, shape and texture characteristics of image comparison, according to the similarity between the statistical characteristics of image evaluation [5]. Neural network image recognition technology is the modern computer technology, image processing, artificial intelligence, pattern recognition theory, developed a kind of new image recognition technology, is in the traditional image recognition method based on fusion of neural network algorithm is a method of image recognition. In text image processing as an example, the character image preprocessing is designed to make character images more clear, the edge is more obvious, and every character segmentation for feature extraction. For this article selects the character images, image preprocessing, the general flow chart is shown in figure 2.

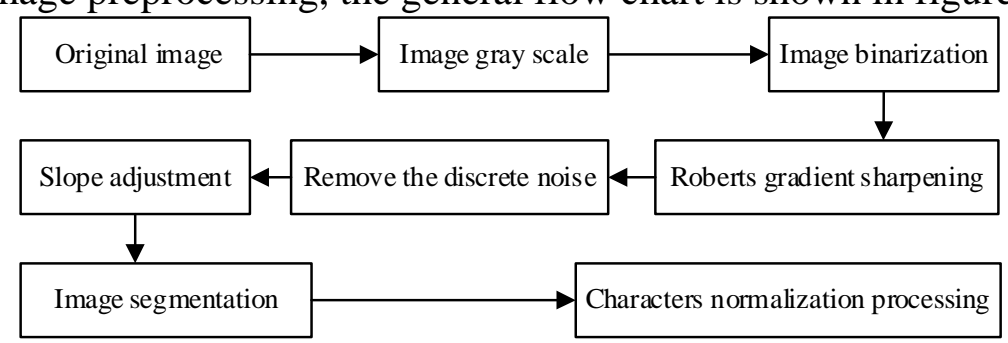

Figure 2. Flow chart of character image pre-processing 
Feature extraction can be thought of as a special kind of image data simplification, its purpose is to extract the high-dimensional data contains the main information of the low dimensional data effectively, is advantageous for the image segmentation and recognition. In general, should have the characteristics of a good distinguishability, good reliability, and independence and less number of these four characteristics. The traditional feature extraction method based on image frequency domain characteristics, including Fourier transform, wavelet transform algorithm, etc. Research in this paper to identify the images, to get the image after preprocessing for the Euler number of feature extracting, character image matrix of each row and each column black pixels to the sum of these two characteristics.

\section{The intelligent image recognition application based on neural network}

This paper, it is by using neural network to distortion image recognition are studied, first with a CCD camera to identify target image information, collected in the process, change the orientation of the camera, collected distortion image information of the most likely target, the target information distortion as far as possible fully, the goals of all kinds of distortion of image information, make up the sample library of target recognition. Then, the sample in the library image is converted to a digital image input mode - number for computer, digital filtering processing, will be some noise and filter out unnecessary information. Finally, the digital information input sample image design for training the neural network learning, generate image recognition system of neural network. Recognition, image with CCD camera's image recognition, after mold - digital conversion, filtering processing input to identify the network system, the system will be real-time calculated results. This paper mainly studied the rotational distortion image recognition, as shown in figure 3 for the 36 samples images of character ' $A$ ', numbered 0 to 35 , each sample image pixels is $40 \mathrm{x} 40$, and in turn clockwise $10^{\circ}$.

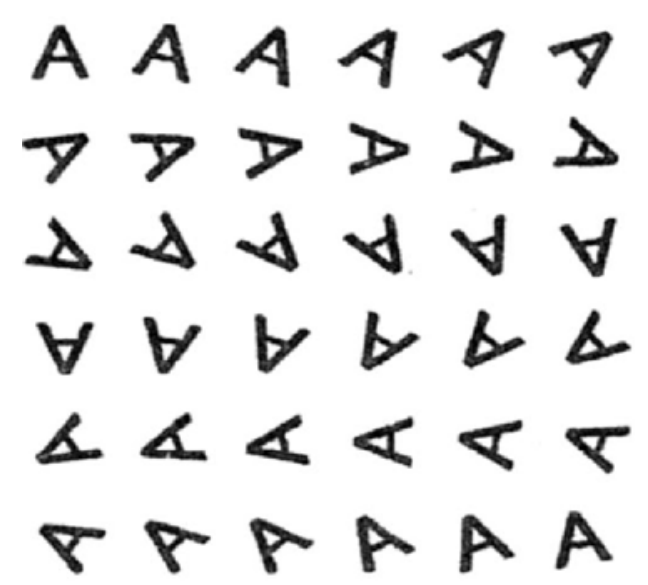

Figure 3. 36 sample images of character ' $A$ '

According to the requirements of identification, the design of three layers BP neural network, the number of hidden layer units according to the improved BP formula $\mathrm{n}^{\prime}=\sqrt{m+n}+a$ to select which $\mathrm{m}$ for the output neuron number, number $\mathrm{n}$ for the output neurons, $\mathrm{a}$ is an integer between 1 -10, determine the structure of the network of $1600 * 45 * 36$;After the normalization of image information processing input network By combining the additional momentum and adaptive rate of the improved BP algorithm, the intermediate transfer function as Tansig and Logsig function, training target error was 0.001 , momentum constant 0 . 8 , the initial vector adaptive for 0 . 01 , vector to increase the ratio of 1.05 , reduce the ratio of 0.7 . Training process is shown in figure 4. 

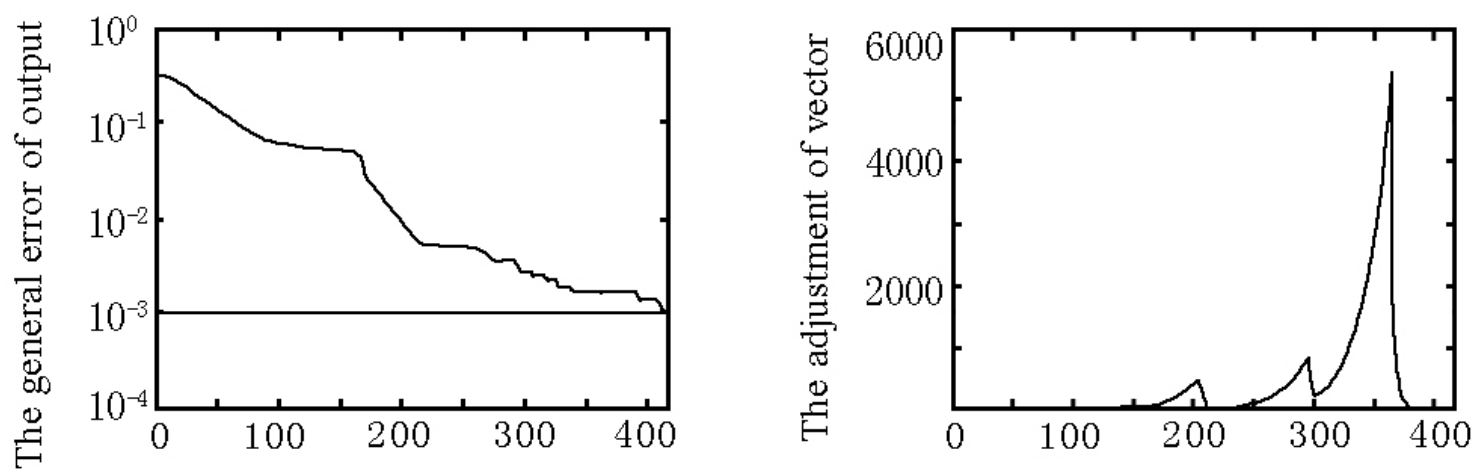

Figure 4. Training process of neural network

For illustrative purposes, this paper only chose 36 sample image in the experiments, the actual work, to improve the recognition accuracy, can choose the 360 samples of every 10 or more, especially for some goals to the distortion of the regularity of the limited time, can include all of its distortion in the training sample, for the modern computer processing speed, tens of thousands of samples can also be a good treatment and calculation, such as adding different proportion in the sample scale, the distortion of image, also can achieve the goal of highly positioning. Accurate recognition and direction of the target, high positioning for industrial production, scientific research and has very important significance in such aspects as national security.

\section{Conclusion}

In this paper, neural network is used to implement the identification of rotational distortion image. In order to improve the training speed of neural network and increase the reliability of the network, by using the momentum and learning rate adaptive adjustment of the improved algorithm of combining the momentum method reduces the sensitivity of the network for the local details of error curved surface, effectively suppress the network in a local minimum, enhances the precision of target identification; The adaptive learning rate adjustment effectively shortens the training time. The experimental results show that image recognition method based on neural network is effective and feasible, with the development of computer technology and artificial intelligence theory, the image recognition technology in target tracking, cruise control, intelligent instrument, robot vision, and other fields will have wide development and application prospect.

\section{References}

[1] G. Hinton, L. Deng, and D. Yu: Signal Processing Magazine, Vol. 29 (2012) No.6, p.82.

[2] E.S.A. Dahshan, T. Hosny, and A.B.M. Salem: Digital Signal Processing, Vol. 20 (2010) No.2, p.433.

[3] M. Karabatak, M.C. Inc: Expert Systems with Applications, Vol. 36 (2009) No.2, p.3465.

[4] O. Mendoza, P. Melin, and G. Licea: Information Sciences, Vol. 179 (2009) No.13, p.2078.

[5] S. Ji, W. Xu, and M. Yang: Pattern Analysis and Machine Intelligence, Vol. 35 (2013) No.1, p.221. 